tosis with amegakaryocytic thrombocytopenia. Am J Hum Genet 2015;97:848-54.

7. Germeshausen M, Ancliff P, Estrada J, et al. MECOM-associated syndrome: a heterogeneous inherited bone marrow failure syndrome with amegakaryocytic thrombocytopenia. Blood Adv 2018;2:586-96.

8. Juskewitch JE, Norgan AP, De Goey SR, et al. How do I $\cdots$ manage the platelet transfusion-refractory patient? Transfusion 2017;57: 2828-35.

9. Nogues N, Garcia A, Julia MR, et al. Anti-HPA15 (gov) antibodies detected in two cases of neonatal alloimmune trombocytopenia and in one case of immune refractoriness to platelet transfusion. Vox Sang 2006;91(Suppl 2):16-17.

10. Gibson BE, Todd A, Roberts I, et al. Transfusion guidelines for neonates and older children. Br J Haematol 2004;124:433-53.

\section{Coagulopathy profiles and their correlation with molecular viral burden in patients with COVID-19}

TO THE EDITOR: Viral infections cause coagulopathy by activating the coagulation cascade. Coagulopathy can result in thrombotic or hemorrhagic complications and plays a central role in determining prognosis [1]. Platelet numbers and functions are also affected during viral infections through various mechanisms. Viruses have been shown to induce thrombocytopenia by affecting platelet production and through peripheral destruction of platelets via specific glycoprotein antibodies [2].

Patients infected with severe acute respiratory syndrome coronavirus 2 (SARS-CoV-2) have shown intrinsic procoagulant effects and inflammatory responses [3]. The underlying mechanisms are still unclear, but apparently, the response against SARS-CoV-2 infection does not differ from infections with previously described coronaviruses [3]. Noticeable thrombotic phenomena with prolonged prothrombin time, activated partial thromboplastin time and increased D-dimer and fibrinogen concentrations have been observed in severe SARS-CoV-2 infections [4]. Here, we report the correlation between clinical coagulopathy profiles and SARS-CoV-2 viral load quantitated by real-time reverse transcriptase-polymerase chain reaction (RT-PCR) in patients with non-severe coronavirus disease 2019 (COVID-19).

A total of 24 patients diagnosed with SARS-CoV-2 infection using real-time RT-PCR were enrolled from February-July 2020 at the Chungbuk National University Hospital (CBNUH), a tertiary hospital in South Korea. Serial blood samples were obtained, and complete blood cell counts were measured using a Beckman DXH 800 (Beckman Coulter, Inc., Fullerton, CA, USA). Coagulation tests including prothrombin time (PT), activated partial thromboplastin time (aPTT), fibrinogen, fibrinogen degradation products (FDP), and D-dimer were performed using a Sysmex CS5100 automatic coagulation analyzer (Siemens Healthcare Diagnostics, Erlangen, Germany). Serial nasal and pharyngeal swabs and/or sputum samples were tested for SARS-CoV-2 using real-time RT-PCR (Allplex 2019-nCoV assay, Seegen, Seoul, Korea). A sample was considered SARS-CoV-2-positive when the $\mathrm{Ct}$ value for the three target genes $(E, R d R p$, and $N)$ was below 40 . The test results were considered inconclusive if one or two of the three genes showed $\mathrm{Ct}$ value below 40 for previously confirmed cases only. A sample was considered SARS-CoV-2-negative when the $\mathrm{Ct}$ value of the three target genes was above 40. Statistical analyses were performed using IBM SPSS Statistics (version 19.0, IBM Corp., Armonk, NY, USA). The Mann-Whitney test was used to analyze the variables between the two groups. Statistical significance was set at $P<0.05$.

Twenty-four patients presented to the CBNUH with confirmed SARS-CoV-2 infection. The median time interval from COVID-19 diagnosis to admission was 3.0 (0-18) days. All patients tested positive for SARS-CoV-2 by real-time RT-PCR. The median age of the cohorts was 55.5 (25-90 $\mathrm{yr})$, and $38 \%(\mathrm{~N}=9)$ were men. A total of 185 nasal and pharyngeal swabs and/or sputum samples were collected

Table 1. Clinical characteristics and laboratory findings in 24 patients diagnosed with COVID-19.

\begin{tabular}{|c|c|}
\hline Variable & Results \\
\hline Age $(y r)^{a)}$ & $55.5(25-90)$ \\
\hline Male sex $(\mathrm{N}, \%)$ & 9/24 (38\%) \\
\hline \multicolumn{2}{|l|}{ Combined disease } \\
\hline Hypertension & $3 / 24(13 \%)$ \\
\hline Diabetes & $4 / 24(17 \%)$ \\
\hline Chronic renal failure & $1 / 24(4 \%)$ \\
\hline $\begin{array}{l}\mathrm{N} \text { of days until molecular negative } \\
\text { conversion }^{\text {b) }}\end{array}$ & $30(16-40)$ \\
\hline \multicolumn{2}{|l|}{ Laboratory findings on admission day ${ }^{c}$} \\
\hline Hemoglobin (g/dL) & $13.1(10.1-16.1)$ \\
\hline White blood cells $\left(\times 10^{3} / \mu \mathrm{L}\right)$ & $4.30(2.24-7.44)$ \\
\hline Neutrophils $\left(\times 10^{3} / \mu \mathrm{L}\right)$ & $2.50(1.11-5.87)$ \\
\hline Lymphocytes $\left(\times 10^{3} / \mu \mathrm{L}\right)$ & $1.15(0.49-1.83)$ \\
\hline Platelets $\left(\times 10^{3} / \mu \mathrm{L}\right)$ & $206(107-554)$ \\
\hline Prothrombin time (INR) & $1.0(0.92-1.2)$ \\
\hline Activated partial thromboplastin time (s) & $29.1(21.7-34.2)$ \\
\hline $\begin{array}{l}\text { Peak fibrinogen }(\mathrm{mg} / \mathrm{dL}) \text { during } \\
\text { hospitalization }\end{array}$ & $482.4(230.4-863.2)$ \\
\hline $\begin{array}{l}\text { Peak D-dimer }(\mathrm{ng} / \mathrm{mL}) \text { during } \\
\text { hospitalization }\end{array}$ & $0.5(0.2-27.1)$ \\
\hline Peak CRP (mg/dL) during hospitalization & $2.9(0.1-17.8)$ \\
\hline Outcome: survival & $24 / 24(100 \%)$ \\
\hline \multicolumn{2}{|c|}{$\begin{array}{l}\text { a) Continuous value represent the median (min-max). }{ }^{\text {b) }} \text { Ten of the } \\
24 \text { patients were discharged after the asymptomatic period lasted } \\
\text { more than } 10 \text { days before the molecular negative conversion. } \\
\text { c) Complete blood count, prothrombin time, and activated partial } \\
\text { thromboplastic time results were described for } 20 \text { of the } 24 \\
\text { patients. The levels of fibrinogen, D-dimer, and CRP were } \\
\text { described in } 14,16 \text {, and } 22 \text { out of } 24 \text { patients, respectively. }\end{array}$} \\
\hline
\end{tabular}


during the infection. There were 85 SARS-CoV-2-positive samples, 61 inconclusive, and 39 negative samples. The clinical characteristics and laboratory findings are summarized in Table 1. Of the 24 patients, 20 had no underlying disease, three had hypertension and diabetes; among these three patients, one also had chronic renal failure. The other patient had only diabetes. The coagulation test of the patients diagnosed with diabetes was initially within the normal range, and there were no definitive findings of thrombosis during hospitalization. The median white blood cells count, absolute neutrophil count, and absolute lymphocyte count, taken on admission day, were $4.3(2.2-7.4) \times 10^{3} / \mu \mathrm{L}, 2.50(1.11-$ $5.87) \times 10^{3} / \mu \mathrm{L}$, and $1.15(0.49-1.82) \times 10^{3} / \mu \mathrm{L}$, respectively. The median hemoglobin level was $13.1(10.1-16.1) \mathrm{g} / \mathrm{dL}$, and the platelet count was $206(107-554) \times 10^{3} / \mu \mathrm{L}$. A decrease in absolute lymphocyte number was observed during the period of infection in which real-time RT-PCR tests were positive, and lymphopenia (less than $1.00 \times 10^{3} / \mu \mathrm{L}$ ) occurred in $10(41.7 \%)$ patients. Neutrophilia $\left(>7.50 \times 10^{3} / \mu \mathrm{L}\right)$ was not observed. Mild thrombocytopenia (less than $150 \times 10^{3} / \mu \mathrm{L}$ ) was observed during the real-time RT-PCR-positive period in six (25\%) patients, and thrombocytosis $\left(>400 \times 10^{3} / \mu \mathrm{L}\right)$ was observed during the real-time RT-PCR-inconclusive period of infection in four (16.7\%) patients.

The real-time RT-PCR results changed from positive to inconclusive and then turned negative. In terms of PT and aPTT, mild prolongation was observed during the positive period of infection towards the upper limit of the reference value and gradually returned to the reference value as it recovered. The absolute lymphocyte levels decreased significantly initially. C-reactive protein (CRP) and fibrinogen significantly increased during the real-time RT-PCR-positive phase and gradually decreased as did markers of the early inflammatory phase (Fig. 1). In contrast, FDP and $\mathrm{D}$-dimer levels gradually increased and peaked during the real-time RT-PCR-inconclusive period of infection. LDH remained persistently high during the real-time RT-PCR-positive and -inconclusive stages, while ESR remained high during all stages.

The COVID-19 pandemic could overwhelm the public healthcare systems. Therefore, laboratory markers to predict disease severity and prognosis are needed to prepare for prolonged periods of sustained infections. Data on previous viral infections showed that coagulation-related parameters and inflammatory markers strongly correlate with the patient's prognosis.
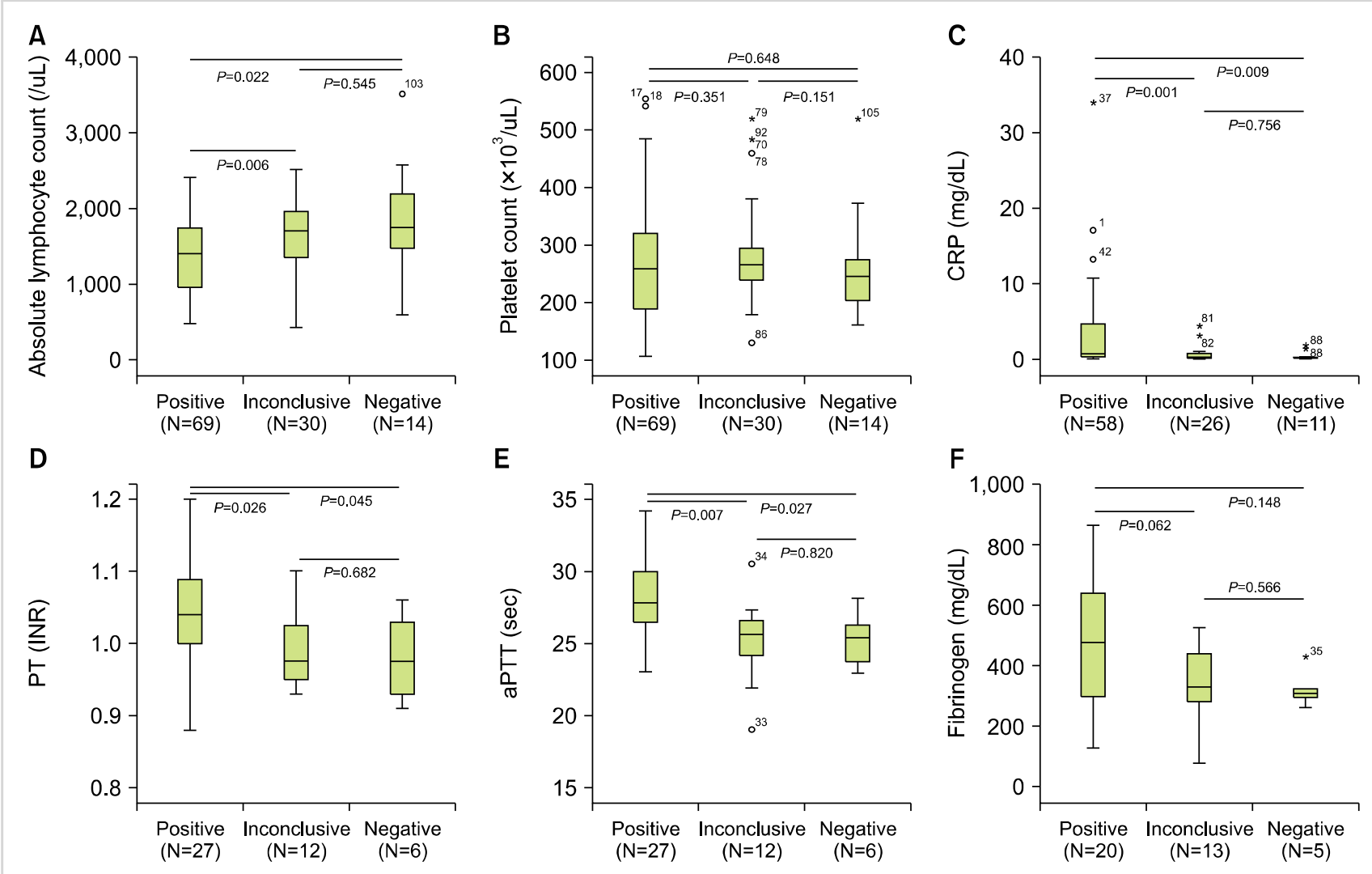

Fig. 1. Changes in coagulopathy-related parameters and inflammation marker over time after confirmation of SARS-CoV-2 infection. Shown are absolute lymphocyte count (A), platelet count (B), CRP (C), PT (D), aPTT (E), fibrinogen (F). The median is shown as a line across the box. The box stretches from the lower 25 th percentile to the upper 75 th percentile. The minimum value is above the lower inner fence, and the maximum value is the inner fence. The symbol ' $O$ ' means that a value is outside the inner fence. The symbol '*' means that a value is outside the outer fence. Abbreviations: aPTT, activated partial thromboplastin time; CRP, C-reactive protein; PT, prothrombin time. 
SARS-CoV-2 has clinical symptoms similar to SARS-CoV-1 and belongs to the same subgenus Sarbecovirus [5]. The immune response to these viral infections is regarded as a general reaction. The pathogenesis of the viruses may differ, but the clinical course and laboratory findings are expected to be similar [6]. In previous SARS-CoV-1 infections, thrombocytopenia has been commonly observed in patients with severe disease [7]. In the case of the Middle East respiratory syndrome (MERS), anemia, lymphopenia, and thrombocytopenia are common, but severe thrombocytopenia is rare $[8,9]$. Disseminated intravascular coagulation is a major cause of death in both SARS-CoV-1 and MERS infections. In our study, thrombocytopenia and lymphopenia were not commonly found. However, since our patients did not have a severe infection, these conditions may differ depending on the disease severity. Further analyses are required in this regard.

Our data show that negative molecular seroconversion takes longer in SARS-CoV-2 infection (median duration of 30 days), and this finding is in line with a previous report [10]. SARS-CoV-2 RNA persists longer than SARSCoV-1, which seroconverts back to negative after 10-15 days of symptom onset [11]. These viral clearance times are important for the establishment of public policies aimed at controlling COVID-19 dissemination. During the real-time RT-PCR-inconclusive infection period, patients were asymptomatic, and we were uncertain as to whether patient isolation should continue. However, hypercoagulability mostly occurred during this phase. In addition, during the real-time RT-PCR-inconclusive phase, test results were discrepant depending on the type of specimen or laboratory method employed [12]. Therefore, patient discharge should be done cautiously.

This study has a few limitations. The small sample size may not truly reflect viral infection characteristics in every patient infected with SARS-CoV-2. However, given that the clinical data on hematological abnormalities in patients with SARS-CoV-2 infection were limited, the results of this study add new information regarding COVID-19. Furthermore, the current study differs from other studies in that it describes coagulopathy-related laboratory results relative to the viral burden. A second limitation is that this study did not include patients with severe COVID-19. This is due to the low mortality rate in South Korea, despite the occurrence of several large clusters [13].

In conclusion, we have shown coagulopathy-related laboratory findings in patients with non-severe COVID-19. The coagulopathy-related laboratory findings indicate that the coagulation profile should be used to monitor patients until negative conversion for SARS-CoV-2 occurs. However, the study's limitations highlight the need for further research focusing specifically on disease severity.
Hee Sue Park ${ }^{1,2}$, Kyeong Seob Shin ${ }^{2}$, Bo Ra Son ${ }^{2}$ ${ }^{1}$ Department of Laboratory Medicine, Chungbuk National University Hospital, ${ }^{2}$ Department of Laboratory Medicine, Chungbuk National University College of Medicine, Cheongju, Korea

Correspondence to: Bo Ra Son Department of Laboratory Medicine, Chungbuk National University College of Medicine, 1 Chungdaero, Seowon-Gu, Cheongju 28644, Korea E-mail: brson@cbungbuk.ac.kr

Received on Aug. 20, 2020; Revised on Nov. 2, 2020; Accepted on Feb. 1, 2021 https://doi.org/10.5045/br.2021.2020210

\section{Authors' Disclosures of Potential Conflicts of Interest}

No potential conflicts of interest relevant to this article were reported.

\section{REFERENCES}

1. Goeijenbier M, van Wissen M, van de Weg C, et al. Review: viral infections and mechanisms of thrombosis and bleeding. J Med Virol 2012;84:1680-96.

2. Assinger A. Platelets and infection - an emerging role of platelets in viral infection. Front immunol 2014;5:649.

3. Connors JM, Levy JH. COVID-19 and its implications for thrombosis and anticoagulation. Blood 2020;135:2033-40.

4. Li Q, Cao Y, Chen L, et al. Hematological features of persons with COVID-19. Leukemia 2020;34:2163-72.

5. Lu R, Zhao X, Li J, et al. Genomic characterisation and epidemiology of 2019 novel coronavirus: implications for virus origins and receptor binding. Lancet 2020;395:565-74.

6. Terpos E, Ntanasis-Stathopoulos I, Elalamy I, et al. Hematological findings and complications of COVID-19. Am J Hematol 2020; 95:834-47.

7. Yang M, Ng MH, Li CK. Thrombocytopenia in patients with severe acute respiratory syndrome (review). Hematology 2005;10:101-5.

8. Kim ES, Choe PG, Park WB, et al. Clinical progression and cytokine profiles of Middle East respiratory syndrome coronavirus infection. J Korean Med Sci 2016;31:1717-25.

9. Joob B, Wiwanitkit V. Magnitude to thrombocytopenia among the patients with novel Middle East respiratory syndrome. Platelets 2015;26:612.

10. Carmo A, Pereira-Vaz J, Mota V, et al. Clearance and persistence of SARS-CoV-2 RNA in patients with COVID-19. J Med Virol 2020;92:2227-31.

11. Perlman S, Dandekar AA. Immunopathogenesis of coronavirus infections: implications for SARS. Nat Rev Immunol 2005;5: 917-27.

12. Liu WD, Chang SY, Wang JT, et al. Prolonged virus shedding even after seroconversion in a patient with COVID-19. J Infect 2020;81:318-56.

13. Shim E, Tariq A, Choi W, Lee Y, Chowell G. Transmission potential and severity of COVID-19 in South Korea. Int J Infect Dis 2020;93:339-44. 